\title{
Structure Analysis of Two Toxoplasma gondii and Neospora caninum Satellite DNA Families and Evolution of Their Common Monomeric Sequence
}

\author{
Marina Clemente, ${ }^{1}$ Natalia de Miguel, ${ }^{2}$ Veronica V. Lia, ${ }^{3}$ Mariana Matrajt, ${ }^{4}$ Sergio O. Angel ${ }^{2,5}$ \\ ${ }^{1}$ INGEBI, Ciudad de Buenos Aires, Argentina \\ ${ }^{2}$ Laboratorio de Parasitologia Molecular, UB2, IIB-INTECH, CONICET-UNSAM, Camino de Circunvalacion Laguna Km. 6, C.C 164, \\ (B7130IIWA), Chascomus, Prov. Buenos Aires, Argentina \\ ${ }^{3}$ Departamento de Ecologiá, Genética y Evolución, Facultad de Ciencias Exactas y Naturales, Universidad de Buenos Aires, Argentina \\ ${ }^{4}$ Department of Biology, University of Pennsylvania, Philadelphia, PA 19104, USA \\ ${ }^{5}$ Departamento de Parasitologiá, ANLIS Dr. Carlos G. Malbran, Ciudad de Buenos Aires, Argentina
}

RE: J Mol Evol (2004) 58:557-567. Figures 2, 3, and 5 of this article were presented incorrectly in the print issue, in which the photographic segment of each figure was inadvertently omitted. The figures appear here correctly along with their captions.
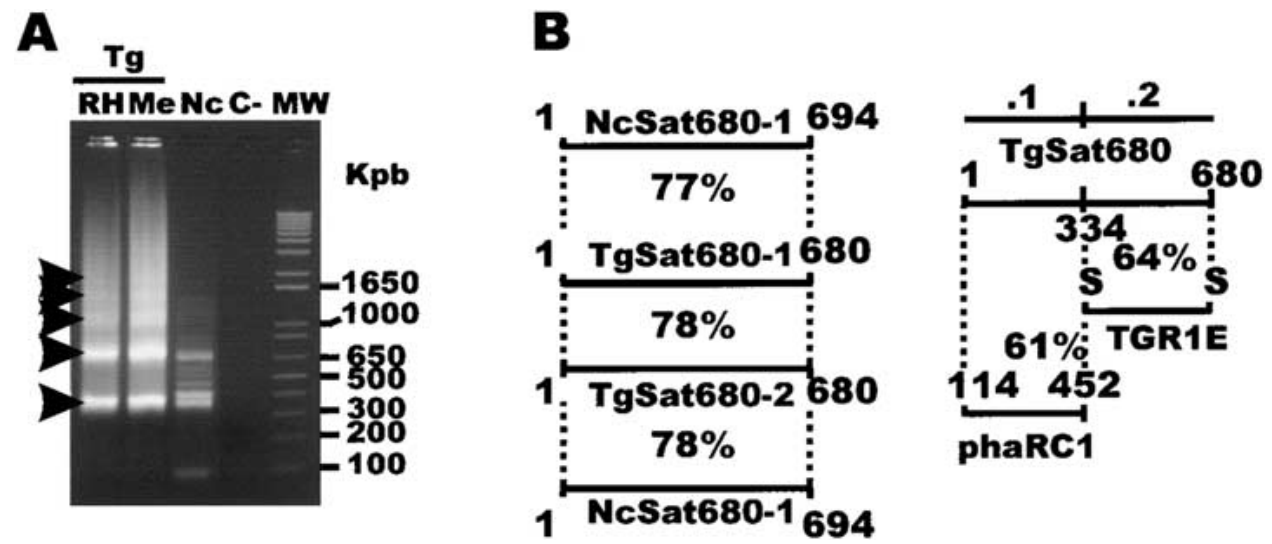

Fig. 2. Cloning of Sat680 element. A Genomic DNA from T. gondii RH (virulent, type I), Me49 (avirulent, type II) strains, and $N$. caninum $(\mathrm{Nc})$ was amplified by PCR with $350 \mathrm{~F} 1$ and $350 \mathrm{R} 2$ primers. The figure shows the PCR products electrophoresed in an agarose gel containing ethidium bromide. C-control without DNA. Arrowheads on the left indicate bands observed in RH and Me49 (Me) lanes that are approximately $350 \mathrm{bp}$, or a multiple of $350 \mathrm{bp}$,

long. Bands of 350- and 680-bp regions from $\mathrm{RH}, \mathrm{Me}$, and Nc were recovered, cloned in pGEM T easy (Promega) vector, sequenced, and deposited in the GenBank database. B Sequence analysis of 680 -bp repetitive elements. Numbers indicate nucleotide positions. The 680-bp elements were split around position 334, giving the halves .1 and .2. Identity percentages were obtained by Blast 2 analysis. 


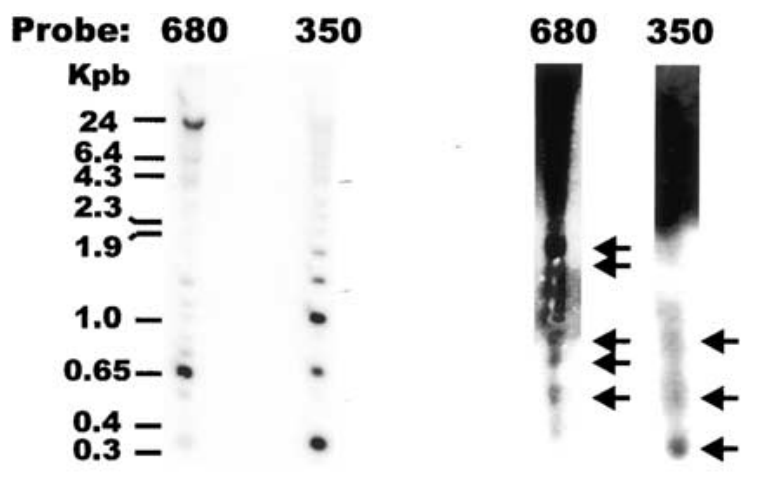

\section{T. gondii N. caninum}

Fig. 3. Tandem organization of Sat 350 and Sat680 elements. DNA from $T$. gondii and $N$. caninum were digested with SalI, blotted on a nylon membrane, and hybridized with ${ }^{32} \mathrm{P}-\mathrm{TgSat} 350-3$ and ${ }^{32} \mathrm{P}$-TgSat $680-2$ probes. T. gondii bands of $\sim 350$ bp or multiples, are clearly observed with Sat 350 probe. Arrows on the right of $N$. caninum Southern blot indicate detected bands. In the case of $T$. gondii DNA hybridization, membranes were exposed for $3 \mathrm{~h}$ in a phosphoimager, whereas in the case of $N$. caninum DNA hybridization membranes were exposed for $48 \mathrm{~h}$ to autoradiographies. 1 $\mathrm{Kpb}$-plus and $\lambda$-HindIII were used as DNA standard (Invitrogen).

A AEIAR GE/GR AE/CR CEIAR

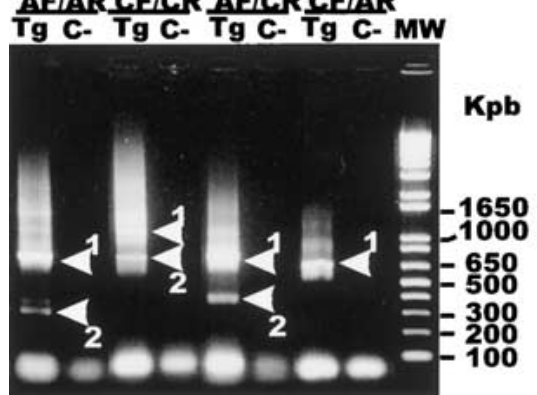

Fig. 5. PCR amplification and cloning of new 350-bp related structures. Genomic DNA from $T$. gondii (A) and $N$. caninum (B) was amplified by PCR with different combinations of AF, AR, CF, and CR primers. The figure shows the PCR products electro-
B

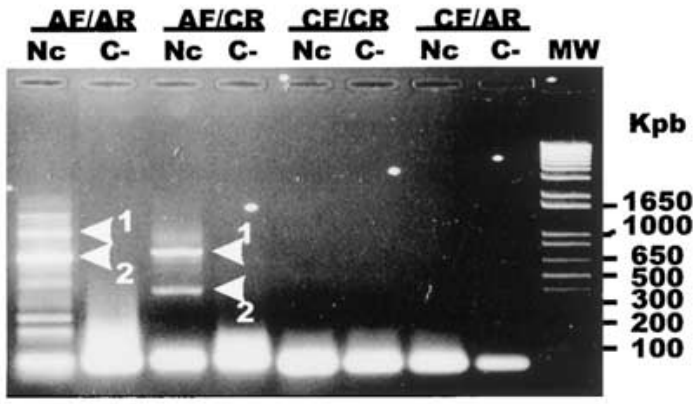

phoresed in an agarose gel containing ethidium bromide. C-control without DNA. Arrows on the right indicate bands recovered from the gel, which were cloned in pGEM T easy (Promega) vector, sequenced, and deposited in the GenBank database. 